\title{
Immunochemotherapy for primary central nervous system lymphoma with rituximab, methotrexate, cytarabine and dexamethasone: Retrospective analysis of 18 cases
}

\author{
JING LIU $^{1 *}$, XUE-FEI SUN $^{1 *}$, JUN QIAN $^{1}$, XUE-YAN BAI $^{1}$, HONG ZHU $^{1}$, QU CUI ${ }^{1}$, \\ XIAO-YAN LI ${ }^{1}$, YUE-DAN CHEN ${ }^{1}$, YA-MING WANG ${ }^{2}$ and YUAN-BO LIU ${ }^{1}$ \\ ${ }^{1}$ Department of Hematology, Beijing Tiantan Hospital, Capital Medical University, Beijing 100050; \\ ${ }^{2}$ Department of Neurosurgery, Navy General Hospital of People's Liberation Army, Beijing 100048, P.R. China
}

Received February 19, 2015; Accepted April 15, 2015

DOI: $10.3892 / \mathrm{mco} .2015 .566$

\begin{abstract}
The incidence of primary central nervous system lymphoma (PCNSL) has increased in the last two decades and the clinical research regarding the treatment for PCNSL patients has also increased. However, the optimal induction chemotherapy has not been fully established. In the present retrospective study, the aim was to analyze the outcome in PCNSL patients treated with the combination of rituximab, methotrexate (MTX), cytarabine (Ara-C) and dexamethasone (R-MAD). Eighteen patients from Beijing Tiantan Hospital (Beijing, China) between January 2010 and March 2014 were newly diagnosed with PCNSL [diffuse large B-cell lymphoma (DLBCL) type] and received R-MAD as first-line treatment. The dosage was as follows: $375 \mathrm{mg} / \mathrm{m}^{2}$ rituximab was administered on day $0,3.5 \mathrm{~g} / \mathrm{m}^{2} \mathrm{MTX}$ was administered on day $1,1 \mathrm{~g} / \mathrm{m}^{2}$ Ara-C was administered on day 2 and $10 \mathrm{mg}$ dexamethasone was administered on days 1-3, every 3 weeks. After 6 cycles, the overall response rate was $94.5 \%$. Ten $(55.6 \%)$ patients achieved complete response (CR), $7(38.9 \%)$ achieved partial response (PR) and $1(5.6 \%)$ had progressive disease (PD). Patients were followed up from the start of the treatment, median 24.2 months (range 6-48). The overall survival (OS) rate was $94.5 \%$ and progression-free survival rate was $94.5 \%$. The median OS was 22 months (95\% confidence interval, 19.4-24.6). The high level of serum lactate dehydrogenase (LDH) concentration
\end{abstract}

Correspondence to: Dr Yuan-Bo Liu, Department of Hematology, Beijing Tiantan Hospital, Capital Medical University, 6 Tiantan Xili, Beijing 100050, P.R. China

E-mail: yuanbol@ccmu.edu.cn

${ }^{*}$ Contributed equally

Key words: primary central nervous system lymphoma, immunochemotherapy, rituximab, retrospective analysis, serum lactate dehydrogenase was associated with a poor outcome. Among 5 patients with an abnormally high LDH concentration, 1 achieved CR, 3 had PR and 1 had PD. None of the patients experienced any grade 4 toxicity. These results indicated that the R-MAD immunochemotherapy regimen is effective in PCNSL patients without serious toxicity. A prospective investigation with more patients should be administered in order to understand the more accurate effect of the regimen.

\section{Introduction}

Primary central nervous system lymphoma (PCNSL) is an aggressive form, confined to the CNS, including the brain, leptomeninges, spinal cord and intraocular structure (1,2). Currently, it is individualized in the new World Health Organization classification of non-Hodgkin's lymphoma (3). Almost 95\% of PCNSL are diffuse large B-cell lymphoma (DLBCL). Although PCNSL shares certain common characteristics with system DLBCL in morphology, it has its own unique features. Its incidence has significantly increased among immunocompetent patients over the last few decades. However, due to the development of highly active antiretroviral therapies, the incidence appears to be decreasing in immunocompromised patients (4).

PCNSLs are sensitive to chemotherapy and radiotherapy, and they are potentially curable tumors. However, until recently, the overall outcome and prognosis for PCNSL patients remains poor, particularly when compared to that of systemic lymphoma. The level of scientific evidence supporting the therapeutic choices for this disease is extremely low and the different opinions on numerous therapeutic aspects results in no consensus regarding the overall strategy $(5,6)$. A previous study has shown that the methotrexate (MTX)-based regimens were more effective compared to high-dose methotrexare (HD-MTX) alone, but the toxicity is serious (7).

In the present study, the characteristics of 18 PCNSL patients were retrospectively analyzed and the efficacy and safety of the rituximab, MTX, cytarabine (Ara-C) and dexamethasone (R-MAD) regimen was examined as first-line treatment in clinical practice. 


\section{Patients and methods}

Clinical characteristics. Between January 2010 and March 2014, 18 patients were newly diagnosed with PCNSL at the Beijing Tiantan Hospital (Beijing, China) and met the inclusion criteria. All the PCNSL patients were immunocompetent patients and they were proven to be DLBCL by pathological examination. The Beijing Tiantan Hospital Ethics Committee approved the study protocol and all the patients provided written informed consent.

Among these patients, there were 11 men and 7 women whose median age was 51 years old (range, 34-83 years, 8 were $<60$ and 10 were $>60$ years old). For the performance status of the patients according to Eastern Cooperative Oncology Group, there were 3 in grade $0-1$ and 15 in grade 2-4 (Table I).

Neuroimaging. On computed tomography (CT) the lesions of PCNSL were hypodense, hyperdense or mixed dense. In general, the shape of the tumor was irregular and the edge was blurred. The lesions should be considered in the differential diagnosis of subacute cerebral infarction and metastases.

On magnetic resonance imaging (MRI), T1-weighted imaging (WI) lesions were usually isointense or hypointense, and T2WI lesions were isointense or hyperintense. The tumor was single in $6(33.3 \%)$ patients and multiple in $12(66.7 \%)$ patients. There were 35 lesions in total. There were $6(17.1 \%)$ lesions in the supratentorial, $28(80.0 \%)$ in the subtentorial and $1(2.9 \%)$ in the lumbar spine. Ten $(55.6 \%)$ PCNSL cases were involved in deep regions of the brain. Enhancing lesions were observed in all the immunocompetent patients with PCNSL (100\%). There were 5 (14.3\%) lesions with enhancement near the meninges (Table II).

Among 18 patients, 6 were examined by ${ }^{18} \mathrm{~F}$-fludeoxyglucose (FDG)-positron emission tomography-CT. ${ }^{18} \mathrm{~F}-\mathrm{FDG}$ uptake values measured by maximum standardized uptake value (SUV) were 7.2-31.9 in 6 PCNSL patients and this value was $\sim 2.5$ times higher than the average SUV in the normal gray matter.

Clinical presentation. The patients' clinical presentation is relevant to the tumor location, including cognitive dysfunction, psychomotor slowing, personality changes and disorientation; and raised intracranial pressure, headache and focal symptoms. Early presentation usually presents a combination of generalized symptoms such as headache, confusion, lethargy and vomiting. Visual symptoms are typically blurred vision or floaters.

Treatment. The R-MAD as first-line treatment for 18 patients was $375 \mathrm{mg} / \mathrm{m}^{2}$ rituximab administered on day $0 ; 3.5 \mathrm{~g} / \mathrm{m}^{2}$ MTX administered on day 1, intravenous infusion in $3 \mathrm{~h}$; $1 \mathrm{~g} / \mathrm{m}^{2}$ Ara-C administered on day 2 and $10 \mathrm{mg}$ dexamethasone administered on day 1-3, every 3 weeks. To decrease the side effects of MTX, calcium folinate salvage was started after $24 \mathrm{~h}$ of MTX administration every $6 \mathrm{~h}$ for a total of 8 times. When the cerebrospinal fluid (CSF) was involved, intrathecal injection with either $10 \mathrm{mg}$ MTX plus $10 \mathrm{mg}$ dexamethasone, or $50 \mathrm{mg}$ Ara-C plus $10 \mathrm{mg}$ dexamethasone occurred once a week until CSF negative.
Table I. Clinical characteristics of all the patients.

\begin{tabular}{lr}
\hline Characteristics & Patients (n=18) \\
\hline Age, median years (range) & $51(34-83)$ \\
Gender, no. (\%) & \\
Male & $11(61)$ \\
Female & $7(39)$ \\
ECOG, no. (\%) & \\
$0-1$ & $3(17)$ \\
$2-4$ & $15(83)$ \\
IELSG, no. (\%) & \\
$0-1$ & $5(28)$ \\
$2-3$ & $11(61)$ \\
$4-5$ & $2(11)$ \\
LDH, no. $(\%)$ & \\
Normal & $13(72)$ \\
Elevated & $5(28)$ \\
CSF cytology, no. $(\%)$ & \\
Positive & $4(22)$ \\
Negative & $14(78)$ \\
CSF protein, no. $(\%)$ & \\
Normal & $12(67)$ \\
Elevated & $6(33)$ \\
Pathology, no. $(\%)$ & \\
DLBCL & $18(100)$ \\
\hline
\end{tabular}

ECOG, Eastern Cooperative Oncology Group; IELSG, International Extranodal Lymphoma Study Group; LDH, lactate dehydrogenase; CFS, cerebrospinal fluid; DLBCL, diffuse large B-cell lymphoma.

Table II. Examination of MRI.

\begin{tabular}{lc}
\hline MRI & No. $(\%)$ \\
\hline Lesions, $\mathrm{n}$ & \\
$\quad$ Single & $6(33.3)$ \\
Multiple & $12(66.7)$ \\
Strengthen the scan & $18(100.0)$ \\
Deep brain lesions & $10(55.6)$ \\
Lesions location & \\
$\quad$ Supratentorial & $6(17.1)$ \\
Subtentorial & $28(80.0)$ \\
Lumbar spine & $1(2.9)$ \\
Midline shift & $4(22.2)$ \\
Enhancement near the meninges & $5(14.3)$ \\
\hline
\end{tabular}

MRI, magnetic resonance imaging.

Evaluation. According to the curative effect evaluation standard of the International Primary CNS Lymphoma Collaborative Group, patients were assessed after 6 cycles. Complete response (CR), partial response (PR) or progressive 
Table III. Rituximab, methotrexate, cytarabine and dexamethasone (R-MAD).

\begin{tabular}{|c|c|c|c|c|}
\hline Toxicity & Grade 1, no. $(\%)$ & Grade 2, no. (\%) & Grade 3 , no. (\%) & Grade 4, no. $(\%)$ \\
\hline Bone marrow suppression & $6(33.3)$ & $4(22.2)$ & $2(11.1)$ & $0(0.0)$ \\
\hline Gastrointestinal reaction & $2(11.1)$ & $0(0.0)$ & $0(0.0)$ & $0(0.0)$ \\
\hline Liver function damage & $3(16.7)$ & $0(0.0)$ & $1(0.06)$ & $0(0.0)$ \\
\hline Renal function damage & $1(0.06)$ & $0(0.0)$ & $0(0.0)$ & $0(0.0)$ \\
\hline Oral ulcer & $1(0.06)$ & $0(0.0)$ & $1(0.06)$ & $0(0.0)$ \\
\hline
\end{tabular}

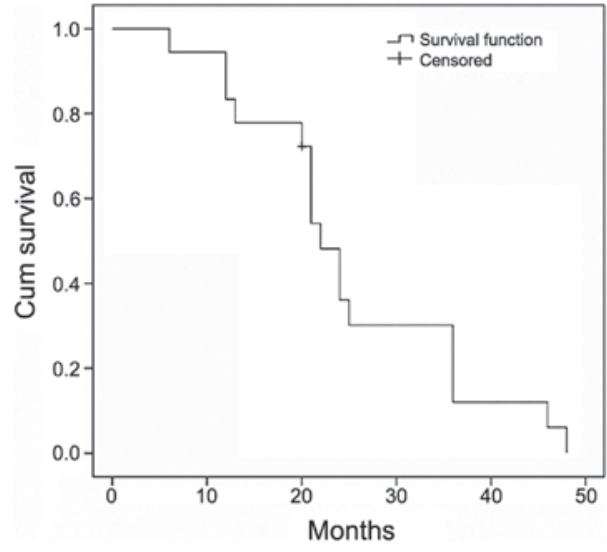

Figure 1. Overall survival rate in the study population.

disease (PD) were defined by MRI. CR was complete disappearance of all the measurable disease and no new lesions. PR was defined as $\geq 50 \%$ reduction of all the measurable lesions. PD was a $25 \%$ increase of measurable lesions or appearance of new lesions. Toxicity was assessed according to the National Cancer Institute-Common Toxicity Criteria (8).

Statistical analysis. Overall survival (OS) was calculated from the start of diagnosis to the time of mortality from any cause or the last follow-up. Progression-free survival (PFS) was calculated from the start of diagnosis to the time of first disease progression or relapse, or fatality resulting from any cause. Time to PCNSL, survival following PCNSL, PSF and OS were estimated according to Kaplan-Meier method. SPSS 18.0 software (SPSS, Inc., Chicago, IL, USA) was used to conduct statistical analysis. $\mathrm{P}<0.05$ was considered to indicate a statistically significant difference.

\section{Results}

Evaluation of patients following immunochemotherapy. Following 6 cycles of the immunochemotherapy, including R-MAD, 10 (55.6\%) PCNSL patients achieved CR, 7 (38.9\%) achieved PR and 1 (5.5\%) achieved PD. The OS rate was $94.5 \%$ and PFS rate was $94.5 \%$ (Fig. 1). The median survival was 22 months (95\% confidence interval, 19.4-24.6). None of the patients experienced any grade 4 bone marrow toxicity.

MRI analysis. MRI following successful treatment of PCNSL showed small enhancing lesions in regions of the initial tumor or surgical manipulation. Complete disappearance of all the measurable disease and no new lesions following treatment were classified as CR. T1 axial, post-gadolinium MRI of PCNSLs at diagnosis (Fig. 2Aa-Da) and a CR after 6 cycles of immunochemotherapy (Fig. 2Ab-Db) were identified. A 42-year-old man exhibited enhancing lesions in the L1-5 vertebral levels of the spinal canal (Fig. 2A). A 68-year-old man and a 63-year-old woman exhibited a solid enhancing lesion involving the right basal ganglia (Fig. 2B and C). A 39-year-old woman exhibited a diffuse involvement of the left temporal lobe and parietal lobe with enhancing masses (Fig. 2D).

Toxicity. Eighteen patients experienced 6 cycles, and none of the patients exhibited serious side effects in the retrospective review. According to the National Cancer Institute-Common Toxicity Criteria, for the toxicity of bone marrow suppression, $6(33.3 \%)$ patients experienced grade $1,4(22.2 \%)$ experienced grade 2 and $2(11.1 \%)$ experienced grade 3 . For the toxicity of gastrointestinal reaction, $2(11.1 \%)$ patients experienced grade 1. For the toxicity of liver function damage, $3(16.7 \%)$ patients experienced grade 1 and $1(0.06 \%)$ experienced grade 3 . For the toxicity of renal function damage, $1(0.06 \%)$ patient experienced grade 1 . For the toxicity of oral ulcer, $1(0.06 \%)$ patient experienced grade 1 and $1(0.06 \%)$ experienced grade 3 . None of the 18 patients experienced grade 4 toxicities and there were no treatment-related mortalities (Table III).

\section{Discussion}

PCNSL is rare and fatal. The prognosis of untreated PCNSL patients is extremely poor and their median survival time is 1.5-3.3 months (9). It was previously demonstrated that the MTX-based protocols could be effective compared with HD-MTX alone, but the overall response rate is poor and the toxicity is serious $(10,11)$.

Pels et al (7) reported the results of a combination of HD-MTX, high-dose of Ara-C (HD-Ara-C), vinca-alkaloids and alkylating agents treated with PCNSL. The CR rate was $61 \%$, PR rate was $10 \%$ and there were $9 \%$ treatment-related fatalities. In a previous trial, 52 patients were treated with the combination of MTX, teniposide, carmustine and methylprednisolone plus intrathecal chemotherapy followed by whole-brain radiation therapy (WBRT) (12). This regimen resulted in an overall response rate of $81 \%$, a 2 -year OS of $69 \%$ and $10 \%$ of patients succumbed. Ferreri et al (13) treated 41 patients with HD-MTX, HD-Ara-C, thiotepa and idarubicin, and subsequent WBRT. The overall response rate 
Aa

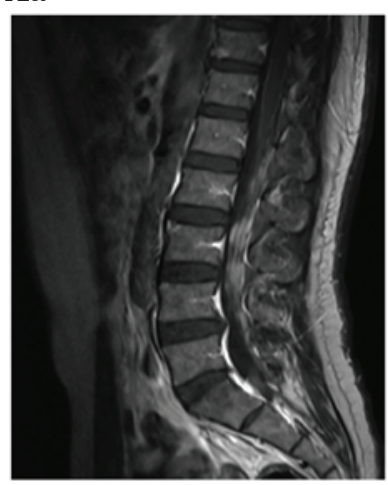

Ba

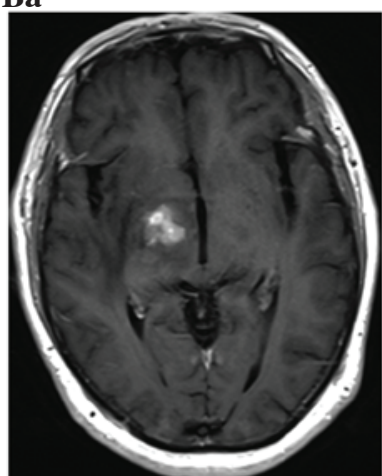

Ca

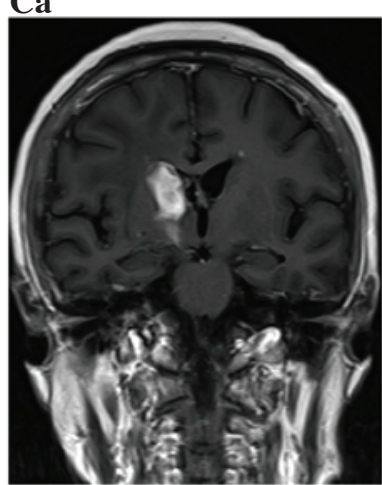

Da

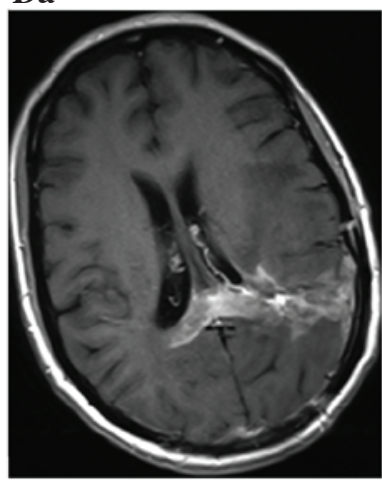

Ab

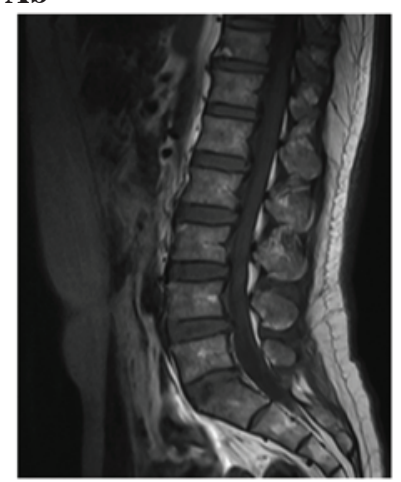

Bb

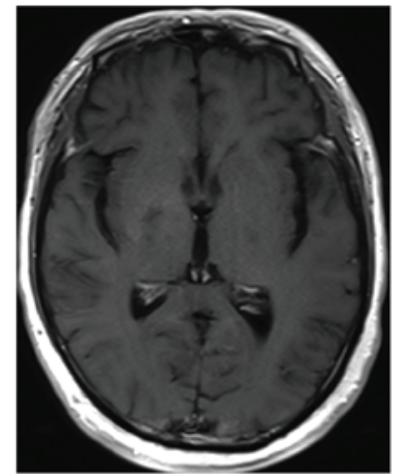

$\mathrm{Cb}$

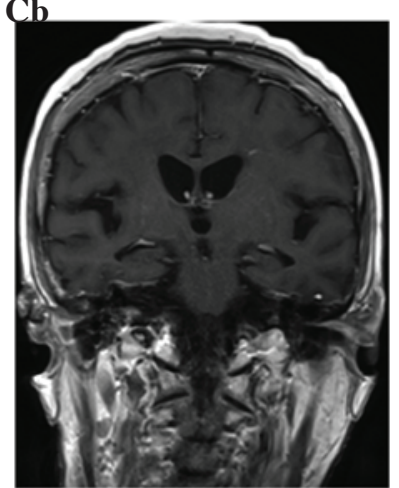

Db

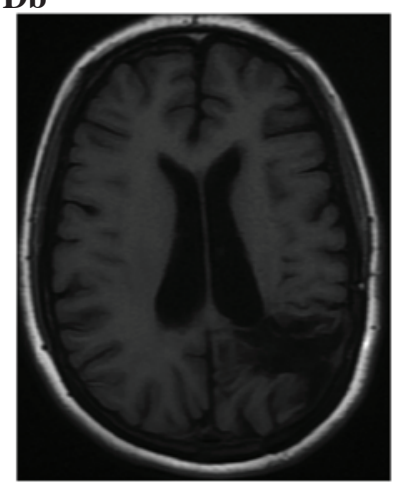

Figure 2. (Aa-Da) T1 axial, post-gadolinium magnetic resonance imaging of primary central nervous system lymphomas at diagnosis and (Ab-Db) at complete response after 6 cycles of immunochemotherapy. (A) Enhancing lesions in the L1-5 vertebral levels of the spinal canal. (B and C) A solid enhancing lesion involving the right basal ganglia. (D) A diffuse involvement of the left temporal lobe and parietal lobe with enhancing masses.

was $83 \%$. In a single-institution study, the overall response rate for HD-MTX plus temozolomide was $70 \%$ and the CR rate was $45 \%$. One treatment-related fatality was observed (14).
In the present study, 18 PCNSL patients were treated with R-MAD, the overall response rate was $94.5 \%$ and none of the patients experienced any grade 4 bone marrow toxicity.

Ara- $\mathrm{C}$ increases the incorporation of cytarabinecytidine triphosphate formation and DNA, which killed more proliferating cells of the S-phase. The combination of MTX and HD-Ara-C increased the rate of CR from 18 to $46 \%(\mathrm{P}=0.006)$. Preliminary results of a randomized phase II study provided evidence for the benefit of the addition of HD-Ara-C to HD-MTX (15). In a large randomized phase II study that evaluated HD-MTX-based induction, with or without HD-Ara-C $\left(2 \mathrm{~g} / \mathrm{m}^{2}\right)$, the median failure-free survival in patients who received the combination of HD-MTX and HD-Ara-C induction was 8 months. By contrast, the median failure-free survival in patients who were administered HD-MTX alone was only 4 months (16). In Western countries, $2 \mathrm{~g} / \mathrm{m}^{2}$ Ara-C was administered twice a day, on days 2 and 3 (15). In the present study, 18 patients received $1 \mathrm{~g} / \mathrm{m}^{2}$ Ara- $\mathrm{C}$ on day 1 in order to decrease the toxicity. None of the patients experienced any grade 4 toxicity. The optimal dose of Ara-C remains to be further studied.

Rituximab is an anti-cluster of differentiation 20 monoclonal antibody. Currently, the addition of rituximab to the cyclophosphamide, doxorubicin, vincristine and prednisone (CHOP) regimen is widely accepted as the standard chemotherapy for systemic DLBCL. Approximately $95 \%$ of PCNSL are DLBCL. However, rituximab is a large protein and has poor penetration to cross the blood-brain barrier (BBB). Addition of rituximab to $\mathrm{CHOP}$ was reported to prevent the CNS dissemination of DLBCL in a retrospective German analysis of 1,222 patients $>60$ years of age, which, however, was not confirmed in a French randomized trial including 399 patients (17). A recent study provided evidence that HD-MTX plus rituximab compared with HD-MTX alone in immunocompetent patients with PCNSL improves the CR and OS rates (18). In CNS lymphoma, rituximab may induce the response of contrast-enhancement lesions, possibly in local where there is disruption of the BBB (19). In the present retrospective study, the addition of rituximab to HD-MTX-based chemotherapy proved feasible in 18 PCNSL patients, with a $\mathrm{CR}$ rate of $55.6 \%$. These results encouraged further research of the precise role of rituximab in the treatment of PCNSL.

Lymphoma cells are sensitive to steroids, however, high-dose steroids cause immunosuppressive effects. In the present retrospective study, we applied short-term and small doses of dexamethasone and achieved an effective outcome.

The serum lactate dehydrogenase (LDH) concentration has been reported to be an independent prognostic marker in PCNSL $(20,21)$. In the present retrospective study, there were 5 patients' LDH concentrations elevated, and 1 of these patients achieved CR, 3 had PR and 1 had PD. These results suggested that the concentration of LDH may be an indicator of poor prognosis.

In conclusion, R-MAD as first-line treatment performs a good effect on PCNSL patients and this regimen is well-tolerated in PCNSL, including elderly patients. Further research of clinical trials is required that focuses on optimization of combination based on high-dose MTX. PCNSL is a rare disease, despite its increasing incidence. The present study was limited by its small sample size and its shorter follow-up 
time. This requires cooperation of multi-center studies and more evidence is required in further research. The poor treatment and prognosis of PCNSL stimulated the requirement to provide the certain pathogenesis and the pathological and biological features of PCNSL, which may help to stratify therapy with specific regimens for patients, and thus, to improve the outcome.

\section{Acknowledgements}

The present study was supported by the National Natural Science Foundation of China General Program (grant no. 81272842).

\section{References}

1. Batchelor T and Loeffler JS: Primary CNS lymphoma. J Clin Oncol 24: 1281-1288, 2006.

2. Abrey LE: Primary central nervous system lymphoma. Curr Opin Neurol 22: 675-680, 2009.

3. Swerdlow SH, Campo E, Harris NL, et al: World Health Organization Classification of tumours. Pathology and genetics of tumours of haematopoietic and lymphoid tissues. WHO press: WHO classification of tumours of haematopoietic and lymphoid tissues. 4th edition. IARC, Lyon, pp236-237, 2008.

4. Corn BW, Marcus SM, Topham A, Hauck W and Curran WJ Jr: Will primary central nervous system lymphoma be the most frequent brain tumor diagnosed in the year 2000? Cancer 79: 2409-2413, 1997.

5. Morris PG and Abrey LE: Therapeutic challenges in primary CNS lymphoma. Lancet Neurol 8: 581-592, 2009.

6. Nayak L and Batchelor TT: Recent advances in treatment of primary central nervous system lymphoma. Curr Treat Options Oncol 14: 539-552, 2013.

7. Pels H, Schmidt-Wolf IG, Glasmacher A, Schulz H, Engert A, Diehl V,Zellner A, Schackert G, Reichmann H, Kroschinsky F, et al: Primary central nervous system lymphoma: Results of a pilot and phase II study of systemic and intraventricular chemotherapy with deferred radiotherapy. J Clin Oncol 21: 4489-4495, 2003.

8. Abrey LE, Batchelor TT, Ferreri AJM, Gospodarowicz M, Pulczynski EJ, Zucca E, Smith JR, Korfel A, Soussain C, DeAngelis LM, et al; International Primary CNS Lymphoma Collaborative Group: Report of an international workshop to standardize baseline evaluation and response criteria for primary CNS lymphoma. J Clin Oncol 23: 5034-5043, 2005.

9. Reni M, Ferreri AJ, Garancini MP and Villa E: Therapeutic management of primary central nervous system lymphoma in immunocompetent patients: Results of a critical review of the literature. Ann Oncol 8: 227-234, 1997.

10. Cobert J, Hochberg E, Woldenberg $\mathrm{N}$ and Hochberg F. Monotherapy with methotrexate for primary central nervous lymphoma has single agent activity in the absence of radiotherapy: a single institution cohort. J Neurooncol 98: 385-393, 2010.
11. Ferreri A J, Reni M, Foppoli M, et al; International Extranodal Lymphoma Study Group (IELSG): High-dose cytarabine plus high-dose methotrexate versus high-dose methotrexate alone in patients with primary CNS lymphoma: a randomised phase 2 trial. Lancet 374: 1512-1520, 2009.

12. Poortmans PM, Kluin-Nelemans HC, Haaxma-Reiche $H$, Van't Veer M, Hansen M, Soubeyran P, Taphoorn M, Thomas J, Van den Bent M, Fickers M, et al; European Organization for Research and Treatment of Cancer Lymphoma Group: High-dose methotrexate-based chemotherapy followed by consolidating radiotherapy in non-AIDS-related primary central nervous system lymphoma: European Organization for Research and Treatment of Cancer Lymphoma Group Phase II Trial 20962. J Clin Oncol 21: 4483-4488, 2003.

13. Ferreri AJ, Dell'Oro S, Foppoli M, Bernardi M, Brandes AA, Tosoni A, Montanari M, Balzarotti M, Spina M, Ilariucci F, et al: MATILDE regimen followed by radiotherapy is an active strategy against primary CNS lymphomas. Neurology 66: 1435-1438, 2006

14. Wang XX, Huang HQ, Bai B, Cai QQ, Cai QC, Gao Y, Xia YF, Xia ZJ and Jiang WQ: Clinical outcomes of patients with newly diagnosed primary central nervous system lymphoma are comparable on treatment with high-dose methotrexate plus temozolomide and with high-dose methotrexate plus cytarabine: A single-institution experience. Leuk Lymphoma 55: 2497-2501, 2014.

15. Ferreri AJM, Reni M, Foppoli M, et al: Randomized phase II trial on primary chemotherapy with high-dose methotrexate alone or associated with highdose cytarabine for patients with primary CNS lymphoma (IELSG \#20 Trial): Tolerability, activity and event-free survival analysis. Blood 112: 580, 2008.

16. Ferreri AJ, Reni M, Foppoli M, Martelli M, Pangalis GA, Frezzato M, Cabras MG, Fabbri A, Corazzelli G, Ilariucci F, et al; International Extranodal Lymphoma Study Group (IELSG): High-dose cytarabine plus high-dose methotrexate versus high-dose methotrexate alone in patients with primary CNS lymphoma: A randomised phase 2 trial. Lancet 374: 1512-1520, 2009.

17. Feugier P, Virion JM, Tilly H, Haioun C, Marit G, Macro M, Bordessoule D, Recher C, Blanc M, Molina T, et al: Incidence and risk factors for central nervous system occurrence in elderly patients with diffuse large-B-cell lymphoma: Influence of rituximab. Ann Oncol 15: 129-133, 2004.

18. Holdhoff M, Ambady P, Abdelaziz A, Sarai G, Bonekamp D, Blakeley J, Grossman SA and Ye X: High-dose methotrexate with or without rituximab in newly diagnosed primary CNS lymphoma. Neurology 83: 235-239, 2014.

19. Batchelor TT, Grossman SA, Mikkelsen T, Ye X, Desideri S and Lesser GJ: Rituximab monotherapy for patients with recurrent primary CNS lymphoma. Neurology 76: 929-930, 2011.

20. Ghesquières H, Drouet Y, Sunyach MP, Sebban C, Chassagne-Clement C, Jouanneau E, Honnorat J, Biron P and Blay JY: Evidence of time-dependent prognostic factors predicting early death but not long-term outcome in primary CNS lymphoma: A study of 91 patients. Hematol Oncol 31: 57-64, 2013.

21. Liu BL, Cheng JX, Zhang X, Zhang W and Cheng H: Limited role of surgery in the management of primary central nervous system lymphoma (Review). Oncol Rep 22: 439-449, 2009. 